\title{
Blocking Caspase Activity Prevents Transsynaptic Neuronal Apoptosis and the Loss of Inhibition in Lamina II of the Dorsal Horn after Peripheral Nerve Injury
}

\author{
Joachim Scholz, ${ }^{1}$ Daniel C. Broom, ${ }^{1}$ Dong-Ho Youn, ${ }^{1}$ Charles D. Mills, ${ }^{1}$ Tatsuro Kohno, ${ }^{1}$ Marc R. Suter, ${ }^{2}$ \\ Kimberly A. Moore, ${ }^{1}$ Isabelle Decosterd, ${ }^{2}$ Richard E. Coggeshall, ${ }^{3}$ and Clifford J. Woolf ${ }^{1}$ \\ ${ }^{1}$ Neural Plasticity Research Group, Department of Anesthesia and Critical Care, Massachusetts General Hospital and Harvard Medical School, Charlestown, \\ Massachusetts 02129, ${ }^{2}$ Anesthesiology Pain Research Group, Department of Anesthesiology, Lausanne University Hospital and Institute of Cell Biology and \\ Morphology, Faculty of Biology and Medicine, Lausanne University, 1005 Lausanne, Switzerland, and ${ }^{3}$ Department of Anatomy and Neuroscience, \\ University of Texas Medical Branch, Galveston, Texas 77555-1069
}

We show that transsynaptic apoptosis is induced in the superficial dorsal horn (laminas I-III) of the spinal cord by three distinct partial peripheral nerve lesions: spared nerve injury, chronic constriction, and spinal nerve ligation. Ongoing activity in primary afferents of the injured nerve and glutamatergic transmission cause a caspase-dependent degeneration of dorsal horn neurons that is slow in onset and persists for several weeks. Four weeks after spared nerve injury, the cumulative loss of dorsal horn neurons, determined by stereological analysis, is $>20 \%$. GABAergic inhibitory interneurons are among the neurons lost, and a marked decrease in inhibitory postsynaptic currents of lamina II neurons coincides with the induction of apoptosis. Blocking apoptosis with the caspase inhibitor benzyloxycarbonyl-Val-Ala-Asp(OMe)-fluoromethylketone (zVAD) prevents the loss of GABAergic interneurons and the reduction of inhibitory currents. Partial peripheral nerve injury results in pain-like behavioral changes characterized by hypersensitivity to tactile or cold stimuli. Treatment with zVAD, which has no intrinsic analgesic properties, attenuates this neuropathic pain-like syndrome. Preventing nerve injury-induced apoptosis of dorsal horn neurons by blocking caspase activity maintains inhibitory transmission in lamina II and reduces pain hypersensitivity.

Key words: caspase-3; glutamate excitotoxicity; GABA; spinal inhibition; dorsal horn; neuropathic pain

\section{Introduction}

Caspases are a family of proteases that selectively hydrolyze peptide bonds on the carboxyl end of an aspartate residue (Degterev et al., 2003). Caspases play a key role in mediating apoptosis caused by activation of death receptors, disturbance of mitochondrial function, and endoplasmatic reticulum stress (Ferri and Kroemer, 2001; Degterev et al., 2003). Apoptotic pathways initiated by these diverse events ultimately converge on the cleavage and activation of the "executioner" protease, caspase-3. In the adult CNS, caspase activation is detected after ischemic brain damage and in animal models of Alzheimer's disease, Huntington's disease, Parkinson's disease, or amyotrophic lateral sclerosis (Yuan and Yankner, 2000; Friedlander, 2003; Tatton et al., 2003). Strategies to improve neuronal survival include targeting up-

Received April 18, 2005; revised June 22, 2005; accepted June 23, 2005.

This work was supported by a Feodor-Lynen fellowship from the Alexander von Humboldt-Foundation (J.S.), National Institute of Neurological Disorders and Stroke (NINDS) Grant NS045459 (C.D.M.), National Institutes of Health National Research Service Award NS11076 (K.A.M.), the Swiss National Science Foundation (I.D.), NINDS Grant NS10161 (R.E.C.), and NINDS Grant NS038253 (C.J.W.). We thank Tarek A. Samad for technical advice, Gena L. Krannig for assistance, and Charles B. Berde for providing the bupivacaine-loaded microspheres.

Correspondence should be addressed to Joachim Scholz, Neural Plasticity Research Group, Department of Anesthesia and Critical Care, Massachusetts General Hospital and Harvard Medical School, 149 13th Street, Room 4309, Charlestown, MA 02129. E-mail: scholz.joachim@mgh.harvard.edu.

DOI:10.1523/JNEUROSCI.1526-05.2005

Copyright $\odot 2005$ Society for Neuroscience $\quad$ 0270-6474/05/257317-07\$15.00/0 stream mediators of caspase activation and direct inhibition of these enzymes (Friedlander, 2003; Benn and Woolf, 2004). Synthetic peptides such as benzyloxycarbonyl-Val-Ala-Asp(OMe)fluoromethylketone (zVAD), which share the recognition motif of caspase substrates, block the activity of caspases competitively and, depending on their linkage to halomethylketones, irreversibly (Ekert et al., 1999). Peptide-based caspase inhibitors reduce neurodegeneration in models of cerebral ischemia, Huntington's disease, and amyotrophic lateral sclerosis (Friedlander, 2003).

Peripheral nerve injury produces apoptosis in the dorsal horn of the spinal cord (Sugimoto et al., 1990; Azkue et al., 1998; Whiteside and Munglani, 2001; Moore et al., 2002). In the superficial dorsal horn (laminas I-III), interneurons containing the transmitters GABA and glycine produce presynaptic and postsynaptic inhibition (Malcangio and Bowery, 1996). After partial peripheral nerve injuries, afferent-evoked IPSCs in lamina II neurons are either abolished or markedly decreased as a result of a reduced presynaptic release of GABA (Moore et al., 2002). Based on the temporal coincidence of apoptotic cell death and the reduction of inhibitory currents after nerve injury, we have hypothesized that apoptosis of inhibitory interneurons leads to a decrease in spinal inhibition and, thereby, to neuropathic pain (Scholz and Woolf, 2002).

We now show that nerve injury-induced apoptosis in the dor- 
sal horn is slow in onset and occurs at low frequency but persists for weeks. The apoptosis is triggered by primary afferent input, mediated by glutamatergic transmission, and involves caspase- 3 activation in neurons. The cumulative loss of dorsal horn neurons is substantial and includes GABAergic interneurons. Caspase inhibition reduces the number of apoptotic profiles, prevents the decrease in spinal inhibition in lamina II, and diminishes pain-like behavior.

\section{Materials and Methods}

Animals. We used adult male Sprague Dawley rats (Charles River Laboratories, Wilmington, MA) for all experiments. Animal procedures were approved by the Subcommittee on Research Animal Care of Massachusetts General Hospital.

Nerve injury models. Surgery was performed under 3\% isoflurane anesthesia. For spared nerve injury (SNI), we ligated the tibial and common peroneal branches of the left sciatic nerve with 5-0 silk and transected them distally (Decosterd and Woolf, 2000). Chronic constriction injury (CCI) consists of four loosely tied 4-0 chromic gut sutures around the proximal sciatic nerve (Bennett and Xie, 1988). For spinal nerve ligation (SNL), the L5 spinal nerve was tightly ligated (Kim and Chung, 1992). For sham operations, the sciatic nerve was exposed at its trifurcation without injury.

Sciatic nerve block. Microspheres loaded with 75\% w/w bupivacaine (BUP) were mixed into Tissucol (Baxter, Volketswil, Switzerland) fibrin sealant (300 mg/ml BUP). A silicone tube (length, $12 \mathrm{~mm}$ ) was incised longitudinally, wrapped around the proximal sciatic nerve, filled with $100 \mu \mathrm{l}$ of the BUP microspheres in fibrin, and sealed with thrombin (Suter et al., 2003). After recovery of the animals, we ensured by nociceptive stimulation, proprioceptive and motor tests that the nerve block was complete. Vehicle treatment consisted of a silicone tube filled with fibrin sealant.

Intrathecal administration of drugs. For single injections of muscimol (Sigma-Aldrich, St. Louis, MO) or zVAD (MP Biomedicals, Irvine, CA), we inserted polyethylene catheters (PE-10) in the lumbar subarachnoid space with the catheter tip positioned dorsally at spinal level L3. We implanted osmotic minipumps (Alzet, Cupertino, CA) subcutaneously for continuous delivery of $500 \mathrm{ng} / \mathrm{h} \mathrm{zVAD}$ ( $\mathrm{Li}$ et al., 2000), dizocilpine (MK-801; $10 \mathrm{nmol} / \mathrm{h}$; Sigma-Aldrich), or vehicle and connected them to an intrathecally placed catheter. Continuous drug treatment started at the time of nerve injury. Animals treated with MK- 801 received an additional bolus injection of MK-801 ( $1 \mathrm{mg} / \mathrm{kg}$, i.p.) before the pump implantation. After completion of the experiments, we confirmed the location of the catheter tip. Animals with neurological deficits after catheter implantation were excluded.

Detection of apoptosis. After transcardial perfusion with $4 \%$ paraformaldehyde, we dissected the spinal cord and collected serial transverse cryosections $(10 \mu \mathrm{m})$ of the L4 and L5 segments. We used the ApopTag Fluorescein In Situ kit (Chemicon, Temecula, CA) to identify apoptotic nuclei by terminal deoxynucleotidyl transferase-mediated X-dUTP nick end labeling (TUNEL) and chromatin staining with bisbenzimide (Hoechst 33342; Chemicon) and counted apoptotic profiles in laminas I-III of the dorsal horn on samples of 10 sections selected in a uniform random manner. We immunolabeled neurons using a monoclonal antibody against neuron-specific nuclear protein (NeuN; 1:1000; Chemicon). Active caspase- 3 was stained with a polyclonal antibody (1:500; Cell Signaling Technology, Beverly, MA).

Disector counts. For total neuron counts, we perfused animals with $1.25 \%$ glutaraldehyde and $1 \%$ paraformaldehyde. We stained four uniform random vibratome sections $(100 \mu \mathrm{m})$ of the $\mathrm{L} 4$ spinal cord freefloating, using peroxidase-antiperoxidase immunohistochemistry for NeuN (1:2000). Stained vibratome sections were embedded in a mixture of Epon and Araldite and sectioned at $1 \mu \mathrm{m}$ thickness. Photographs of two $1 \mu \mathrm{m}$ sections (reference and look-up) separated by three intermediate sections were used to construct montages. The midpoints of lamina II-III and III-IV boundaries were connected with a straight line. Because the sciatic nerve projects medially in the L4 dorsal horn, we counted
NeuN-immunolabeled neurons in the medial half of laminas I-III as delineated previously (Coggeshall et al., 2001).

For counts of glutamic acid decarboxylase 67 (GAD67) mRNApositive neurons, we perfused animals with $4 \%$ paraformaldehyde. In situ hybridization was performed on five random pairs of adjacent cryosections $(10 \mu \mathrm{m})$ through the L4 dorsal horn using a digoxygenin-labeled RNA transcript of a linearized plasmid, which contains a 460 bp GAD67 cDNA insert. Sections were incubated for $10 \mathrm{~min}$ in $0.125 \%$ acetic anhydride and $0.675 \%$ triethanolamine and equilibrated for $30 \mathrm{~min}$ in hybridization buffer $(50 \mathrm{mmol} / \mathrm{L}$ Tris chloride, $50 \%$ formamide, $5 \mathrm{mmol} / \mathrm{L}$ EDTA, $0.6 \mathrm{~mol} / \mathrm{L} \mathrm{NaCl}, 0.25 \%$ SDS, $1 \times$ Denhardt's solution, $10 \%$ dextran sulfate, $100 \mu \mathrm{g} / \mathrm{ml}$ salmon sperm DNA, and $250 \mu \mathrm{g} / \mathrm{ml}$ yeast tRNA). We incubated the sections with $1 \mu \mathrm{g}$ of antisense RNA probe per milliliter of hybridization buffer for $40 \mathrm{~h}$ at $60^{\circ} \mathrm{C}$. Transcripts in sense orientation served as control. After immunodetection with alkaline phosphatase-conjugated anti-digoxygenin Fab fragments (1:500; Roche, Indianapolis, IN), digital photographs of the dorsal horns were montaged and the number of GAD67 mRNA-positive neurons was determined by physical dissector analysis.

Electrophysiological investigations. Two weeks after SNI, we prepared a transverse slice (thickness, $600-700 \mu \mathrm{m}$ ) of the L4 spinal segment with the left dorsal root attached. Whole-cell patch-clamp recordings from lamina II neurons were performed in the ipsilateral dorsal horn. Afferent-evoked IPSCs and currents induced by muscimol were recorded at a holding potential of $0 \mathrm{mV}$ (Moore et al., 2002).

Behavioral testing. After habituation, we obtained two baseline measures during the week before SNI. Animals were examined for 4-5 weeks after surgery. Animals were placed on an elevated wire grid, and the plantar surface of the hindpaw was stimulated within the territory of the sural nerve. We used a series of ascending von Frey monofilaments to test for mechanical allodynia. The withdrawal threshold was determined as the lowest force that provoked a paw withdrawal at least twice in 10 applications. Mechanical hyperalgesia was examined by pricking the skin with a safety pin and measuring the duration of paw withdrawal. To test for cold allodynia, we applied a drop of acetone to the skin (Decosterd and Woolf, 2000).

Statistics. Investigators were blinded to all treatments in all experiments. Data are presented as mean \pm SEM. We used an unpaired twotailed Student's $t$ test to compare between group differences after SNI. A paired $t$ test was applied to calculate differences between dissector counts in the ipsilateral and contralateral dorsal horn. We performed a repeatedmeasures analysis of the behavioral test results, using a mixed-effects model in which the correlation structure over time was modeled as a first-order autoregressive process. Asterisks in the figures indicate levels of significance $\left({ }^{*} p<0.05 ;{ }^{* *} p<0.01 ;{ }^{* *} p<0.001\right)$.

\section{Results}

\section{Peripheral nerve injury causes caspase-dependent apoptosis of dorsal horn neurons}

TUNEL-positive nuclei appear $1 \mathrm{~d}$ after SNI in the ipsilateral dorsal horn of the spinal cord, manifesting chromatin changes typical of apoptosis: condensation, fragmentation, and margination (Fig. $1 A$ ). The numbers of apoptotic profiles (TUNELpositive with typical chromatin changes) peak on day 7 ( $p<$ 0.001 compared with sham-operated controls) and remain significantly elevated $21 \mathrm{~d}$ after injury (Fig. $1 \mathrm{~B}$ ). The nerve injuryinduced apoptosis is restricted to the ipsilateral dorsal horn; there is no increased occurrence of apoptotic profiles contralaterally. We found more apoptotic nuclei after SNI in the L4 dorsal horn $(14.8 \pm 0.83$ profiles per sample on day 7$)$, in which the majority of sciatic nerve afferents terminate (Swett and Woolf, 1985), than in the L5 dorsal horn $(3.80 \pm 0.59)$ (Fig. $1 C)$. There are no signs of necrosis in Nissl- or Fluoro-Jade B-stained (Schmued and Hopkins, 2000) dorsal horn sections after SNI.

To explore whether the induction of apoptosis involves the executioner caspase- 3 in dorsal horn neurons, we combined immunostaining for cleaved (active) caspase-3 (Casp-3a) and the 
A

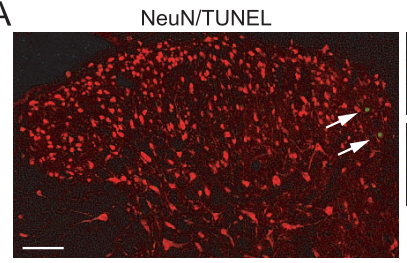

TUNEL H33342 Merged

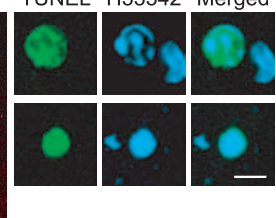

\section{B}

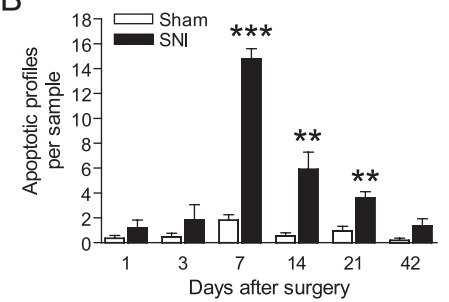

C
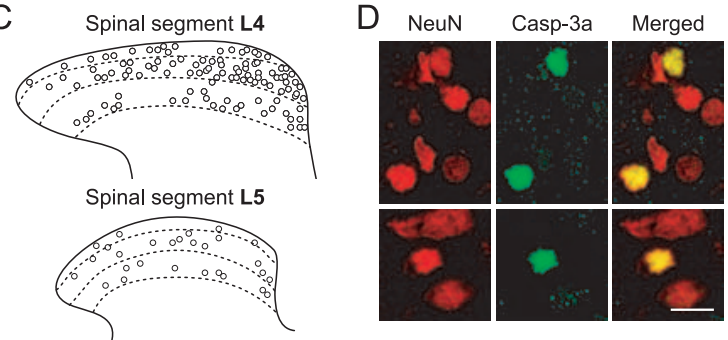

$E$

$\mathrm{F}$

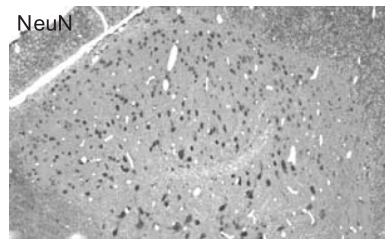

Ipsilater al

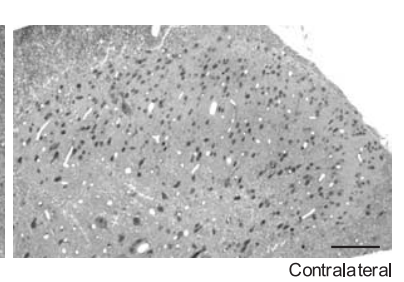

Figure 1. Apoptosis and neuronal loss after SNI. A, Confocal images of TUNEL-positive nuclear profiles in the ipsilateral L4 dorsal horn. High magnification shows fragmentation and condensation of chromatin [stained with bisbenzimide; Hoechst 33342 (H33342)]. B, Numbers of apoptotic profiles peaked on day 7 and were significantly elevated for 3 weeks after injury. C, More apoptotic profiles were found in the $L 4$ dorsal horn compared with the $L 5$ dorsal horn $7 \mathrm{~d}$ after SNI. Overlays from six animals, 10 sections per animal, are shown. $\boldsymbol{D}$, Casp-3a in the nuclei of dorsal horn neurons labeled by NeuN immunostaining. $\boldsymbol{E}$, Continuous intrathecal delivery of zVAD reduced the number of apoptotic profiles in the $L 4$ dorsal horn $7 \mathrm{~d}$ after SNI. $\boldsymbol{F}, \boldsymbol{G}$, Dissector counts revealed a substantial reduction in the number of neurons (NeuN) in the $L 4$ dorsal horn 4 weeks after SNI. $B, E$, $\boldsymbol{G}, n=5-6$. Scale bars: $\boldsymbol{A}$, left, $100 \mu \mathrm{m}$, right, $5 \mu \mathrm{m} ; \boldsymbol{D}, 5 \mu \mathrm{m} ; \boldsymbol{F}, 100 \mu \mathrm{m}$.

neuronal nuclear protein NeuN. Seven days after SNI, we found Casp-3a localized in the nuclei of neurons in the L4 dorsal horn $(4.38 \pm 0.63$ profiles per sample, compared with $0.28 \pm 0.18$ contralaterally; $p<0.001$ ) (Fig. $1 D$ ). Continuous intrathecal administration of the caspase inhibitor $\mathrm{zVAD}$ for $7 \mathrm{~d}$ after the injury reduces the number of apoptotic profiles (Fig. $1 E$ ), indicating that the nerve injury-induced apoptosis of dorsal horn neurons depends on caspase activation.

\section{Injury-induced loss of dorsal horn neurons}

To determine the loss of neurons, we performed a stereological analysis of cells immunopositive for NeuN 4 weeks after SNI, after the injury-provoked apoptosis had declined, and found that the number of neurons in the ipsilateral L4 dorsal horn (laminas I-III) was reduced by $22 \%$ (Fig. $1 F, G$ ). To confirm the reliability and specificity of the neuronal marker, we examined these sections by electron microscopy and found that all cells identified by electron microscopy as neurons $(n=100)$ are NeuN immunoreactive, whereas glial cells $(n=169)$ are not.

\section{Apoptosis is triggered by afferent activity}

A pattern of apoptosis similar to that produced by SNI also occurs in two other models of peripheral nerve injury, CCI of the sciatic nerve and ligation of the L5 spinal segmental nerve (SNL) (Fig. 2). After SNL, we found apoptotic profiles in both the L4 and L5 segments of the dorsal horn (Fig. 2). As in SNI, there is no in-

crease of apoptotic profiles in the contralateral dorsal horn after CCI or SNL (data not shown).

To explore whether afferent activity is required for the induction of apoptosis after SNI, we blocked conduction in the sciatic nerve proximal to the lesion site using a silicone tube filled with BUP-loaded microspheres (Suter et al., 2003) (Fig. 3A). The microspheres produce a sensory and motor block that lasts for 7-8 d. This treatment decreases apoptosis in the L4 dorsal horn (Fig. 3B). However, $7 \mathrm{~d}$ after the end of the BUP block (i.e., 14-15 d after the start of the treatment), the number of apoptotic profiles in the dorsal horn is essentially the same as in vehicle-treated animals $7 \mathrm{~d}$ after SNI (Fig. 3B). Therefore, a temporary peripheral nerve block delays, but does not eliminate, nerve injury-evoked apoptosis, suggesting that ongoing afferent activity is responsible for the protracted induction of apoptosis.

The major excitatory transmitter released by primary sensory afferents that terminate in the superficial dorsal horn is glutamate, which acts on ionotropic and metabotropic receptors (Moore et al., 2000). High-calcium influx after activation of the NMDA glutamate receptor is associated with excitotoxic apoptosis (Hardingham and Bading, 2003). We tested whether nerve injury-induced apoptosis is mediated by NMDA receptor activation using the noncompetitive antagonist MK-801. An intraperitoneal injection of MK-801 (1 mg/kg) at the time of nerve injury followed by continuous intrathecal application $(10 \mathrm{nmol} / \mathrm{h})$ significantly decreased the number of apoptotic profiles $7 \mathrm{~d}$ after SNI (Fig. 3C).

\section{Blocking apoptosis prevents the loss of spinal inhibition}

Peripheral nerve injury causes a reduction in presynaptic GABA release, leading to a decrease in spinal postsynaptic inhibition (Moore et al., 2002).

GAD67 synthesizes GABA and is a marker for GABAergic inhibitory interneurons (Mackie et al., 2003). Using GAD67 mRNA in situ hybridization, we found that, 4 weeks after SNI, the number of GABAergic interneurons in the ipsilateral dorsal horn decreases by $24.2 \pm 2.7 \%$ in laminas I-II and $25.0 \pm 2.5 \%$ in lamina III, compared with the contralateral side $(p<0.001)$. The number of GABAergic neurons in the contralateral dorsal horn is unchanged compared with the dorsal horn of naive rats. To block caspase activity and prevent injury-induced apoptosis of GABAergic neurons, we administered zVAD intrathecally, starting at the time of SNI. Continuous treatment with zVAD for 4 weeks protects against the loss of GAD67 mRNA-positive neurons $(106.1 \pm 11.0 \%$ in lamina I-II and $104.5 \pm 8.2 \%$ in lamina III compared with contralateral) (Fig. 4), indicating that the decrease in GABAergic interneurons results from apoptosis.

To test whether caspase-dependent apoptosis of interneurons is responsible for the reduced postsynaptic inhibition of superficial dorsal horn neurons after nerve injury, we recorded primary afferent-evoked IPSCs in zVAD-treated animals after SNI. In na- 
A

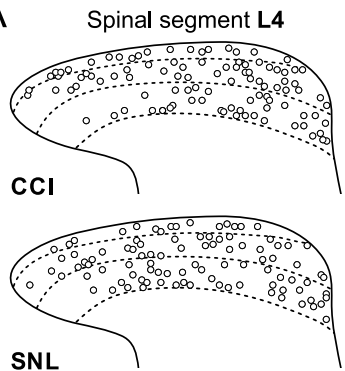

B

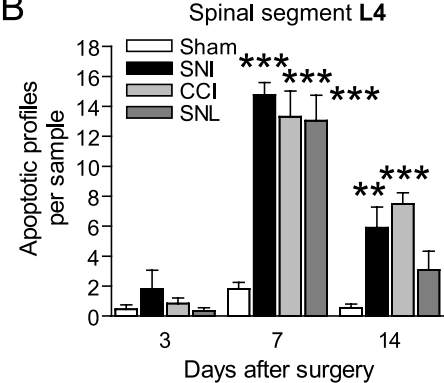



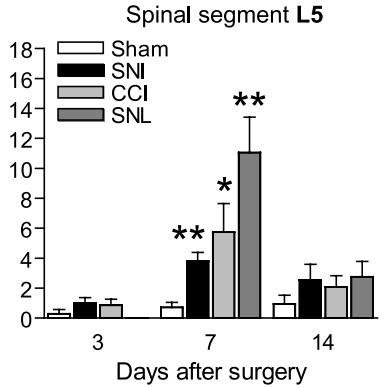

Figure 2. Apoptosis in the dorsal horn induced by CCl and SNL. A, Apoptotic profiles in the ipsilateral $L 4$ and $L 5$ dorsal horns $7 d$ afternerve injury. Overlays from six animals, 10 sections per animal, are shown. $\boldsymbol{B}$, We found similar numbers of apoptotic profiles in the $L 4$ dorsal horn, whereas the level of apoptosis in the $\mathrm{L} 5$ segment was increased after SNL, compared with SNI or CCl.

A

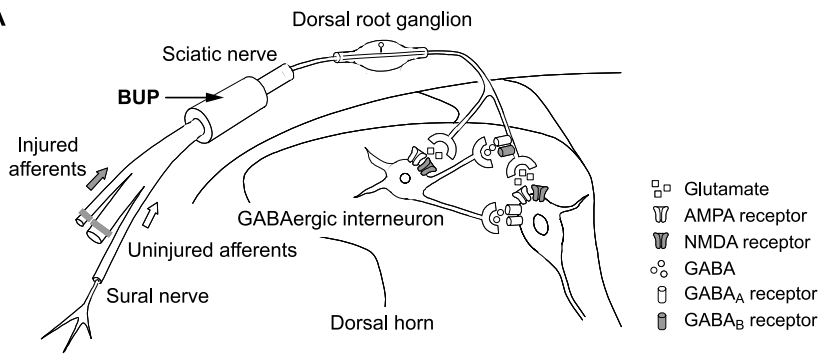

B



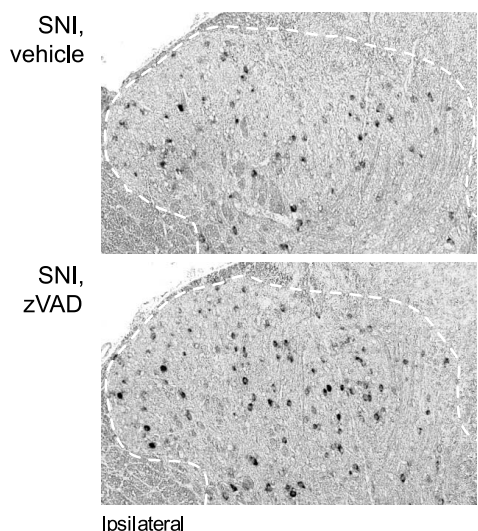



Figure 4. Blocking apoptosis rescued GABAergic interneurons. Continuous treatment with zVAD prevented the loss of GAD67 mRNA-positive neurons in the ipsilateral $L 4$ dorsal horn 4 weeks after SNI. Scale bar, $100 \mu \mathrm{m}$.

line) (Yoshimura and Nishi, 1995; Moore et al., 2002) (Fig. 5B). Although every recorded neuron still has monosynaptic and polysynaptic excitatory input, the number of neurons with detectable afferent-evoked IPSCs drops by $\sim 30 \% 2$ weeks after SNI (Fig. 5A). In these neurons, the peak amplitude of evoked IPSCs is $58.2 \pm 6.6 \mathrm{pA}$ compared with $122.9 \pm 13.8 \mathrm{pA}$ in naive animals $(p<0.01)$ (Fig. $5 C)$, and the decay time constant declines from $33.4 \pm 2.9$ to $13.4 \pm 1.3 \mathrm{~ms}(p<0.01)$ (Fig. $5 D)$. Recordings in the presence of strychnine $(0.5 \mu \mathrm{mol} / \mathrm{L})$ and bicuculline $(5-10$ $\mu \mathrm{mol} / \mathrm{L}$ ) reveal a particularly marked reduction in the GABAergic IPSC component $(p<0.01)$ (Fig. $5 B, E)$. Continuous intrathecal delivery of zVAD, starting at the time of SNI, prevents the decrease in afferent-evoked IPSCs (Fig. 5A-E) and restores the frequency of spontaneous IPSCs $(2.7 \pm 0.6 \mathrm{~Hz}$ compared with $1.2 \pm 0.4 \mathrm{~Hz}$ in vehicle controls; $p<0.05$ ).

The nerve injury-induced loss of inhibition in the dorsal horn is not a consequence of diminished afferent input to interneurons. The number of primary sensory neurons decreases late $(>8$ weeks) after axotomy (Tandrup et al., 2000). There is no loss of monosynaptic excitatory currents in lamina II neurons (Moore et al., 2002), and polysynaptic afferent input actually increases after SNI (Kohno et al., 2003). Nor does the decrease in afferentevoked IPSCs result from a reduced sensitivity to GABA. In contrast, outward currents of lamina II neurons in response to the $\mathrm{GABA}_{\mathrm{A}}$ receptor agonist muscimol $(5 \mu \mathrm{mol} / \mathrm{L})$ are enhanced after SNI ( $p<0.001$ ) (Fig. 5F). We found that muscimol administration does not elicit inward currents in any neuron recorded in lamina II. Intrathecal injections of muscimol attenuate the hypersensitivity to mechanical stimuli after SNI $(p<0.05)$ (Fig. $5 G$ ), indicating that $\mathrm{GABA}_{\mathrm{A}}$ receptors in the dorsal horn are functional and produce analgesia.

Figure 3. Nerve injury-induced apoptosis depends on glutamatergic afferent input. $\boldsymbol{A}$, Schematic drawing illustrating the sciatic nerve block proximal to the lesion site. $B, A$ continuous conduction block produced by BUP decreased the number of apoptotic profiles on day 7 after SNI. Seven days after the blockhad worn off, the number of apoptotic profiles was comparable with that in vehicle-treated animals $7 \mathrm{~d}$ after SNI. C, Continuous blockade of spinal NMDA receptors by intrathecal MK-801 reduced the apoptosis in the dorsal horn $7 \mathrm{~d}$ after SNI $(n=5-6)$.

ive animals, afferent stimulation evokes IPSCs in $>95 \%$ of lamina II neurons (Fig. 5A). These IPSCs have two elements: a glycinergic component (blocked by strychnine) and a GABAergic component (blocked by the $\mathrm{GABA}_{\mathrm{A}}$ receptor antagonist bicucul-

\section{Anti-apoptotic treatment reduces neuropathic pain-like behavior}

The nerve damage associated with SNI, CCI, or SNL elicits behavioral features characteristic of neuropathic pain: hypersensitivity to normally innocuous tactile or cold stimuli (mechanical and cold allodynia) and exaggerated responses to noxious mechanical stimuli (pinprick hyperalgesia) (Bennett and Xie, 1988; Kim and Chung, 1992; Decosterd and Woolf, 2000). Continuous intrathecal delivery of zVAD for 4 weeks after $\mathrm{SNI}$ attenuates the development of mechanical allodynia $(p<0.001$, compared with vehicle treatment), pinprick hyperalgesia $(p<0.01)$, and 
A

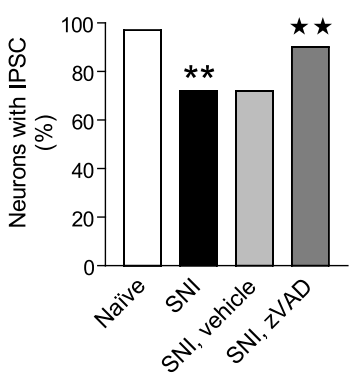

D

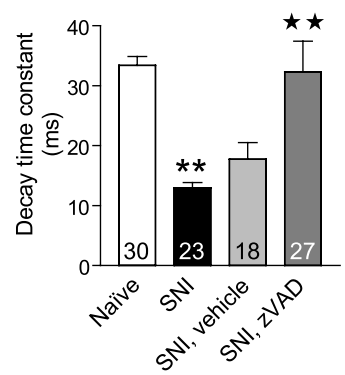

B

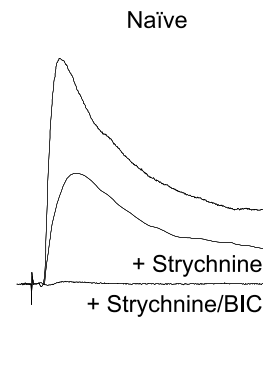

E

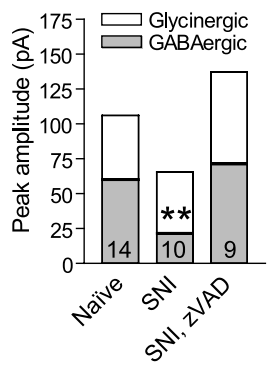

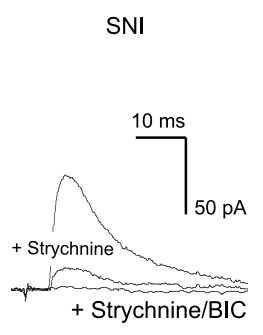



F
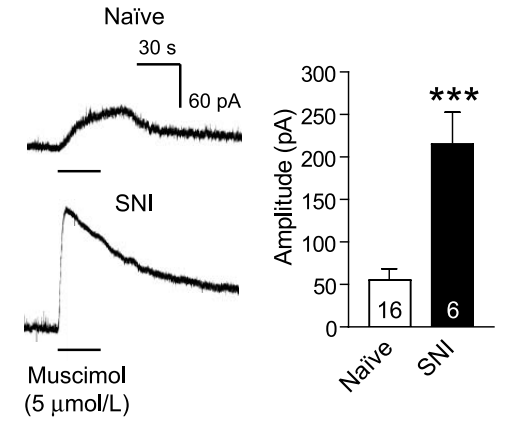

C



G

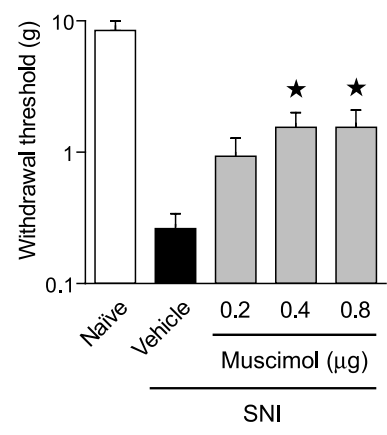

Figure 5. Blocking apoptosis preserved spinal inhibition. A, Continuous zVAD treatment protected against the loss of afferent-evoked IPSCs recorded from lamina II neurons of the ipsilateral L4 dorsal horn 2 weeks after SNI. B, Representative recordings of evoked IPSCS. C, Caspase inhibition by ZVAD prevented the decrease in peak amplitude of remaining IPSCS. Numbers of recorded neurons are shown inside the bars. $\boldsymbol{D}$, Shortening of the decay time constant of the IPSCs reflected a loss of GABAergic currents after SNI and was not seen in recordings from zVAD-treated animals. $\boldsymbol{E}$, IPSC recordings in the presence of strychnine $(0.5 \mu \mathrm{mol} / \mathrm{L})$, followed by bicuculline $(5-10 \mu \mathrm{mol} / \mathrm{L})$, confirmed the decrease in GABAergic currents. $F$, Bath application of the $G_{A B A}$ receptor agonist muscimol elicited potentiated responses of lamina II neurons after SNI. G, Intrathecal administration of muscimol reduced mechanical allodynia, which developed after $\mathbf{S N I}(n=6-7)$. $\boldsymbol{A}, \boldsymbol{C}-\boldsymbol{E}, \boldsymbol{G}$, ${ }^{* *} p<0.01$ compared with naive animals; ${ }^{\star} p<0.05,{ }^{\star \star} p<0.01$ compared with vehicle-treated animals after SNI.
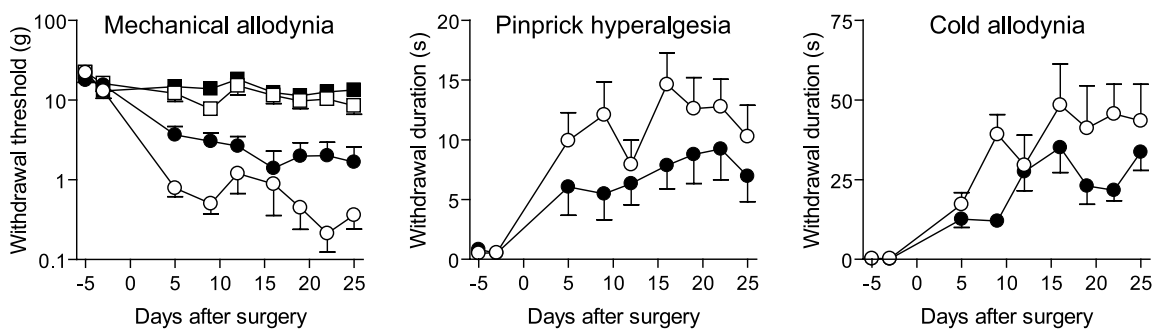

Figure 6. Continuous treatment with zVAD reduced neuropathic pain-like behavior. Blocking caspase-dependent apoptosis attenuated mechanical allodynia ( $p<0.001)$, pinprick hyperalgesia $(p<0.01)$, and cold allodynia $(p<0.01)$ after SNI. $O$, Vehicle ipsilateral; , zVAD ipsilateral; $\square$, vehicle contralateral; $\square$, zVAD contralateral. $n=8$.

cold allodynia $(p<0.01)$ without altering the sensitivity of the contralateral hindpaw (Fig. 6). Mechanical allodynia (withdrawal threshold, $2.54 \pm 1.30 \mathrm{~g}$ in zVAD-treated animals vs $0.70 \pm 0.32 \mathrm{~g}$ in vehicle controls; not significant) and pinprick hyperalgesia (withdrawal duration, $5.27 \pm 2.57 \mathrm{~s}$ vs $10.46 \pm 3.26 \mathrm{~s} ; p<0.05$ ) remain decreased $5 \mathrm{~d}$ after termination of the zVAD treatment. A single injection of zVAD (500 $\mathrm{ng}$ ) has no effect on basal pain sensitivity in naive animals $(n=6)$ nor does it reduce neuropathic pain-like behavior (mechanical allodynia, pinprick hyperalgesia, cold allodynia) in animals $21 \mathrm{~d}$ after SNI ( $n=6$; data not shown). Blocking apoptosis with a treatment that has no intrinsic analgesic properties, but which prevents the loss of neurons and preserves GABAergic inhibition in dorsal horn lamina II, attenuates neuropathic pain.

\section{Discussion}

Peripheral nerve injury produces apoptosis in the ipsilateral dorsal horn of the spinal cord that leads to a cumulative loss of $>20 \%$ of neurons in superficial laminas over 4 weeks. During this time, caspase- 3 is activated and translocated to the nucleus of dorsal horn neurons. The prolonged induction of cell death over several weeks and the transient nature of apoptotic events prohibit estimating the magnitude of cell loss from apoptotic profile counts (Polgar et al., 2004). As for other chronic neurodegenerative conditions (Yuan and Yankner, 2000), only few apoptotic profiles are detected at any time. In situ markers of apoptosis, such as TUNEL, are positive for a very short time, only 1-3 h (Gavrieli et al., 1992; Rossiter et al., 1996). Using assumption-based profile counts to estimate the number of surviving neurons (Ibuki et al., 1997; Eaton et al., 1998; de Novellis et al., 2004) is also inappropriate: a quantitative evaluation of neuronal loss requires stereological counting techniques (West, 1999). Therefore, we used a physical dissector method to determine the decrease in dorsal horn neurons 4 weeks after SNI. Because of the delayed onset and the extended course of nerve injury-provoked apoptosis, this time interval is necessary to assess the neuronal loss. The protracted nature of neuronal death in the dorsal horn may explain why previous attempts to establish a loss of neurons early after the lesion, within the first 2 weeks, have failed (Polgar et al., 2003, 2004).

To investigate whether neuronal activity after nerve injury is responsible for the induction of apoptosis in the dorsal horn, we produced a temporary conduction block proximal to the injury site. Blocking afferent input prevents the apoptosis but only for the duration of the block. Once the conduction block is removed, apoptosis resumes. The neurodegeneration is obviously not 
caused by the short-lived injury discharge, an intense firing of afferent fibers that follows nerve transection (Liu et al., 2000). Axotomy alone is insufficient to provoke a loss of dorsal horn neurons (Coggeshall et al., 2001) or a decrease in IPSCs (Moore et al., 2002). We show that ongoing afferent activity determines the fate of dorsal horn neurons after partial nerve lesion. We hypothesize that ectopic discharges trigger the activity-induced apoptosis of dorsal horn neurons. Injured afferents and neighboring primary sensory fibers that are not directly affected by the lesion develop ectopic activity a few days after nerve injury, and this spontaneous activity is maintained for weeks (Liu et al., 2000; Michaelis et al., 2000; Wu et al., 2001). Because we see many apoptotic profiles in the L4 spinal segment after an L5 spinal nerve ligation, a large proportion of transsynaptic apoptosis after partial nerve injury is likely provoked by the ectopic activity of neighboring uninjured afferents. Blocking the ionotropic NMDA glutamate receptor reduces nerve injury-induced apoptosis, indicating that excitotoxic levels of glutamate released by primary afferents cause the degeneration of dorsal horn neurons. Presumably by mimicking the effect of ectopic activity, electrical stimulation of a sciatic nerve after complete transection causes substantial neuronal loss (Coggeshall et al., 2001).

Four weeks after SNI, we found a 25\% reduction of GABAergic interneurons labeled by GAD67 mRNA in situ hybridization (Mackie et al., 2003). The total GAD67 protein level in the dorsal horn remains stable after SNI (Moore et al., 2002), implying that surviving interneurons increase the synthesis of GAD67. There are conflicting reports on changes in GABA-immunoreactive cells after peripheral nerve injury, which likely reflect the different timings of nerve lesions, the spinal cord segments analyzed, and the variability of this immunohistochemical technique (Ibuki et al., 1997; Eaton et al., 1998; Polgar et al., 2003). We found that the proportion of lost GABAergic interneurons equals the decline of total neurons, arguing against a selective vulnerability of these interneurons to nerve injury-induced degeneration. Blocking apoptosis by caspase inhibition prevents the decrease in GABAergic interneurons after SNI and preserves the integrity of GABAergic spontaneous and afferent-evoked IPSCs in lamina II. Treatment with zVAD protects from both the complete loss of IPSCs in $\sim 30 \%$ of lamina II neurons and a substantial reduction of IPSCs in the remaining lamina II neurons. These results suggest that caspase activation in the interneurons is responsible for the decrease in spinal inhibition after nerve injury. The fact that the relative reduction of GABAergic inhibition is greater than the loss of GABAergic interneurons indicates a caspase-dependent functional impairment of surviving neurons. Enhanced afferent input resulting from GABAergic disinhibition probably further aggravates the loss of dorsal horn neurons: reducing GABAergic inhibition pharmacologically with bicuculline results in an increased A fiber-mediated polysynaptic excitation of lamina II neurons in naive animals (Baba et al., 2003). A similar recruitment of polysynaptic A fiber input to lamina II cells occurs after nerve injury (Okamoto et al., 2001; Kohno et al., 2003). After peripheral nerve injury, GABA anomalously produces excitation in a subgroup of lamina I neurons as a result of altered anion transport (Coull et al., 2003). Complementary to the loss of inhibition in lamina II neurons, this change may well also increase excitation and pain sensitivity after nerve injury, particularly if present in projection neurons (Mantyh and Hunt, 2004). However, we and others (Malan et al., 2002) have observed that intrathecal administration of GABA agonists decreases painlike behavior after nerve injury, indicating that the predominant
GABAergic effect in the spinal cord is to produce inhibition, not excitation.

Blocking nerve injury-induced apoptosis in the dorsal horn attenuates neuropathic pain-like behavior. Because the onset of apoptosis is slow, it is very unlikely that the degeneration of inhibitory interneurons contributes to the development of pain in the first few days after nerve injury. The effect of caspase inhibition on pain-like behavior becomes evident at the time when apoptosis peaks. The improvement is incomplete, presumably because not all the neurons rescued from apoptosis fully maintain their functional integrity. In addition, other mechanisms that are independent of the degeneration of inhibitory interneurons contribute to neuropathic pain. These include afferent activity-evoked central sensitization (Woolf and Salter, 2000), microglia activation (Tsuda et al., 2005), and altered modulation of sensory transmission by pathways descending from the brainstem (Mantyh and Hunt, 2004; Suzuki et al., 2004). The reduction of neuropathic pain produced by zVAD outlasts its discontinuation, suggesting that degeneration of inhibitory interneurons contributes to the persistence of neuropathic pain. The prolonged effect is certainly not attributable to the half-life of caspase inhibition by zVAD, which is $<40 \mathrm{~min}$ (Garcia-Calvo et al., 1998).

We demonstrate here that glutamate-mediated transsynaptic apoptosis causes a loss of dorsal horn neurons, including GABAergic interneurons, after peripheral nerve injury. There is a major difference between the protracted induction of neurodegeneration in the dorsal horn after nerve injury and the rapid neuronal loss observed in other forms of excitotoxicity. For example, after cerebral ischemia, traumatic brain injury, or epilepsy (Dirnagl et al., 1999; Liou et al., 2003), neurons die immediately either by necrosis or apoptosis, depending on the extent of the excitotoxic insult. A second wave of apoptosis may follow as the vulnerability of neurons in the vicinity of the primary lesion increases or survival conditions deteriorate (Dirnagl et al., 1999; Liou et al., 2003). In contrast, we found no signs of necrosis in the dorsal horn; only apoptotic cell death is observed, with a late peak on day 7 after the lesion. Consequently, the loss of dorsal horn neurons after nerve injury resembles a chronic neurodegenerative condition rather than an acute insult. Neuroprotective treatment strategies that are sustained for the duration of nerve injury-induced apoptosis may offer a novel, disease-modifying approach to the management of chronic neuropathic pain.

\section{References}

Azkue JJ, Zimmermann M, Hsieh TF, Herdegen T (1998) Peripheral nerve insult induces NMDA receptor-mediated, delayed degeneration in spinal neurons. Eur J Neurosci 10:2204-2206.

Baba H, Ji RR, Kohno T, Moore KA, Ataka T, Wakai A, Okamoto M, Woolf CJ (2003) Removal of GABAergic inhibition facilitates polysynaptic A fibermediated excitatory transmission to the superficial spinal dorsal horn. Mol Cell Neurosci 24:818-830.

Benn SC, Woolf CJ (2004) Adult neuron survival strategies-slamming on the brakes. Nat Rev Neurosci 5:686-700.

Bennett GJ, Xie YK (1988) A peripheral mononeuropathy in rat that produces disorders of pain sensation like those seen in man. Pain 33:87-107.

Coggeshall RE, Lekan HA, White FA, Woolf CJ (2001) A-fiber sensory input induces neuronal cell death in the dorsal horn of the adult rat spinal cord. J Comp Neurol 435:276-282.

Coull JA, Boudreau D, Bachand K, Prescott SA, Nault F, Sik A, De Koninck P, De Koninck Y (2003) Trans-synaptic shift in anion gradient in spinal lamina I neurons as a mechanism of neuropathic pain. Nature 424:938-942.

Decosterd I, Woolf CJ (2000) Spared nerve injury: an animal model of persistent peripheral neuropathic pain. Pain 87:149-158. 
Degterev A, Boyce M, Yuan J (2003) A decade of caspases. Oncogene 22:8543-8567.

de Novellis V, Siniscalco D, Galderisi U, Fuccio C, Nolano M, Santoro L, Cascino A, Roth KA, Rossi F, Maione S (2004) Blockade of glutamate mGlu5 receptors in a rat model of neuropathic pain prevents early overexpression of pro-apoptotic genes and morphological changes in dorsal horn lamina II. Neuropharmacology 46:468-479.

Dirnagl U, Iadecola C, Moskowitz MA (1999) Pathobiology of ischaemic stroke: an integrated view. Trends Neurosci 22:391-397.

Eaton MJ, Plunkett JA, Karmally S, Martinez MA, Montanez K (1998) Changes in GAD- and GABA-immunoreactivity in the spinal dorsal horn after peripheral nerve injury and promotion of recovery by lumbar transplant of immortalized serotonergic precursors. J Chem Neuroanat 16:57-72.

Ekert PG, Silke J, Vaux DL (1999) Caspase inhibitors. Cell Death Differ 6:1081-1086.

Ferri KF, Kroemer G (2001) Organelle-specific initiation of cell death pathways. Nat Cell Biol 3:E255-E263.

Friedlander RM (2003) Apoptosis and caspases in neurodegenerative diseases. N Engl J Med 348:1365-1375.

Garcia-Calvo M, Peterson EP, Leiting B, Ruel R, Nicholson DW, Thornberry NA (1998) Inhibition of human caspases by peptide-based and macromolecular inhibitors. J Biol Chem 273:32608-32613.

Gavrieli Y, Sherman Y, Ben Sasson SA (1992) Identification of programmed cell death in situ via specific labeling of nuclear DNA fragmentation. J Cell Biol 119:493-501.

Hardingham GE, Bading H (2003) The yin and yang of NMDA receptor signalling. Trends Neurosci 26:81-89.

Ibuki T, Hama AT, Wang XT, Pappas GD, Sagen J (1997) Loss of GABAimmunoreactivity in the spinal dorsal horn of rats with peripheral nerve injury and promotion of recovery by adrenal medullary grafts. Neuroscience 76:845-858.

Kim SH, Chung JM (1992) An experimental model for peripheral neuropathy produced by segmental spinal nerve ligation in the rat. Pain 50:355-363.

Kohno T, Moore KA, Baba H, Woolf CJ (2003) Peripheral nerve injury alters excitatory synaptic transmission in lamina II of the rat dorsal horn. J Physiol (Lond) 548:131-138.

Li M, Ona VO, Guegan C, Chen M, Jackson-Lewis V, Andrews LJ, Olszewski AJ, Stieg PE, Lee JP, Przedborski S, Friedlander RM (2000) Functional role of caspase- 1 and caspase- 3 in an ALS transgenic mouse model. Science 288:335-339.

Liou AK, Clark RS, Henshall DC, Yin XM, Chen J (2003) To die or not to die for neurons in ischemia, traumatic brain injury and epilepsy: a review on the stress-activated signaling pathways and apoptotic pathways. Prog Neurobiol 69:103-142.

Liu X, Eschenfelder S, Blenk KH, Janig W, Habler H (2000) Spontaneous activity of axotomized afferent neurons after L5 spinal nerve injury in rats. Pain 84:309-318.

Mackie M, Hughes DI, Maxwell DJ, Tillakaratne NJ, Todd AJ (2003) Distribution and colocalisation of glutamate decarboxylase isoforms in the rat spinal cord. Neuroscience 119:461-472.

Malan TP, Mata HP, Porreca F (2002) Spinal GABA(A) and GABA(B) receptor pharmacology in a rat model of neuropathic pain. Anesthesiology 96:1161-1167.

Malcangio M, Bowery NG (1996) GABA and its receptors in the spinal cord. Trends Pharmacol Sci 17:457-462.

Mantyh PW, Hunt SP (2004) Setting the tone: superficial dorsal horn projection neurons regulate pain sensitivity. Trends Neurosci 27:582-584.

Michaelis M, Liu X, Janig W (2000) Axotomized and intact muscle afferents but no skin afferents develop ongoing discharges of dorsal root ganglion origin after peripheral nerve lesion. J Neurosci 20:2742-2748.
Moore KA, Baba H, WoolfCJ (2000) Synaptic transmission and plasticity in the superficial dorsal horn. Prog Brain Res 129:63-80.

Moore KA, Kohno T, Karchewski LA, Scholz J, Baba H, Woolf CJ (2002) Partial peripheral nerve injury promotes a selective loss of GABAergic inhibition in the superficial dorsal horn of the spinal cord. J Neurosci 22:6724-6731.

Okamoto M, Baba H, Goldstein PA, Higashi H, Shimoji K, Yoshimura M (2001) Functional reorganization of sensory pathways in the rat spinal dorsal horn following peripheral nerve injury. J Physiol (Lond) 532:241-250.

Polgar E, Hughes DI, Riddell JS, Maxwell DJ, Puskar Z, Todd AJ (2003) Selective loss of spinal GABAergic or glycinergic neurons is not necessary for development of thermal hyperalgesia in the chronic constriction injury model of neuropathic pain. Pain 104:229-239.

Polgar E, Gray S, Riddell JS, Todd AJ (2004) Lack of evidence for significant neuronal loss in laminae I-III of the spinal dorsal horn of the rat in the chronic constriction injury model. Pain 111:144-150.

Rossiter JP, Riopelle RJ, Bisby MA (1996) Axotomy-induced apoptotic cell death of neonatal rat facial motoneurons: time course analysis and relation to NADPH-diaphorase activity. Exp Neurol 138:33-44.

Schmued LC, Hopkins KJ (2000) Fluoro-Jade B: a high affinity fluorescent marker for the localization of neuronal degeneration. Brain Res 874:123-130.

Scholz J, Woolf CJ (2002) Can we conquer pain? Nat Neurosci 5 [Suppl]:1062-1067.

Sugimoto T, Bennett GJ, Kajander KC (1990) Transsynaptic degeneration in the superficial dorsal horn after sciatic nerve injury: effects of a chronic constriction injury, transection, and strychnine. Pain 42:205-213.

Suter MR, Papaloizos M, Berde CB, Woolf CJ, Gilliard N, Spahn DR, Decosterd I (2003) Development of neuropathic pain in the rat spared nerve injury model is not prevented by a peripheral nerve block. Anesthesiology 99:1402-1408.

Suzuki R, Rygh LJ, Dickenson AH (2004) Bad news from the brain: descending 5-HT pathways that control spinal pain processing. Trends Pharmacol Sci 25:613-617.

Swett JE, Woolf CJ (1985) The somatotopic organization of primary afferent terminals in the superficial laminae of the dorsal horn of the rat spinal cord. J Comp Neurol 231:66-77.

Tandrup T, Woolf CJ, Coggeshall RE (2000) Delayed loss of small dorsal root ganglion cells after transection of the rat sciatic nerve. J Comp Neurol 422:172-180.

Tatton WG, Chalmers-Redman R, Brown D, Tatton N (2003) Apoptosis in Parkinson's disease: signals for neuronal degradation. Ann Neurol 53 [Suppl 3]:S61-S70.

Tsuda M, Inoue K, Salter MW (2005) Neuropathic pain and spinal microglia: a big problem from molecules in "small" glia. Trends Neurosci 28:101-107.

West MJ (1999) Stereological methods for estimating the total number of neurons and synapses: issues of precision and bias. Trends Neurosci 22:51-61.

Whiteside GT, Munglani R (2001) Cell death in the superficial dorsal horn in a model of neuropathic pain. J Neurosci Res 64:168-173.

Woolf CJ, Salter MW (2000) Neuronal plasticity: increasing the gain in pain. Science 288:1765-1769.

Wu G, Ringkamp M, Hartke TV, Murinson BB, Campbell JN, Griffin JW, Meyer RA (2001) Early onset of spontaneous activity in uninjured C-fiber nociceptors after injury to neighboring nerve fibers. J Neurosci 21:RC140(1-5).

Yoshimura M, Nishi S (1995) Primary afferent-evoked glycine- and GABAmediated IPSPs in substantia gelatinosa neurones in the rat spinal cord in vitro. J Physiol (Lond) 482:29-38.

Yuan J, Yankner BA (2000) Apoptosis in the nervous system. Nature 407: 802-809. 